\title{
Impact of Doxycycline as Malaria Prophylaxis on Risk of Influenza-Like Illness among International Travelers
}

\author{
Kathryn Lago, ${ }^{1,2 \star}$ Kalyani Telu, ${ }^{3,4}$ David Tribble, ${ }^{3}$ Anuradha Ganesan, ${ }^{3,4,5}$ Anjali Kunz,, ${ }^{6,7}$ Charla Geist, ${ }^{4,7}$ Jamie Fraser, ${ }^{3,4}$ \\ Indrani Mitra, ${ }^{3,4}$ Tahaniyat Lalani, ${ }^{3,4,8}$ and Heather Yun ${ }^{1,2}$ for the Infectious Disease Clinical Research Program TravMil Study Group \\ ${ }^{1}$ Brooke Army Medical Center, Fort Sam Houston, Texas; ${ }^{2}$ Uniformed Services University of the Health Sciences, Bethesda, Maryland; \\ ${ }^{3}$ Department of Preventive Medicine and Biostatistics, Infectious Disease Clinical Research Program, Uniformed Services University of the Health \\ Sciences, Bethesda, Maryland; ${ }^{4}$ The Henry M. Jackson Foundation for the Advancement of Military Medicine, Inc., Bethesda, Maryland; ${ }^{5}$ Walter \\ Reed National Military Medical Center, Bethesda, Maryland; ${ }^{6}$ Madigan Army Medical Center, Tacoma, Washington; ${ }^{7}$ Landstuhl Regional Medical \\ Center, Landstuhl, Germany; ${ }^{8}$ Naval Medical Center, Portsmouth, Virginia
}

\begin{abstract}
Travelers are often at risk for both influenza-like illness (ILI) and malaria. Doxycycline is active against pathogens causing ILI and is used for malaria prophylaxis. We evaluated the risk factors for ILI, and whether the choice of malaria prophylaxis was associated with ILI. TravMil is a prospective observational study enrolling subjects presenting to military travel clinics. Influenza-like illness was defined as subjective fever with either a sore throat or cough. Characteristics of trip and use of malaria prophylaxis were analyzed to determine association with development of ILI. Poisson regression models with robust error variance were used to estimate relative risk (RR) of ILI. A total of 3,227 trips were enrolled: $62.1 \%$ male, median age of 39 years (interquartile range [IQR] 27,59), median travel duration 19 days (IQR 12, 49); $32 \%$ traveled to Africa, $40 \%$ to Asia, and $27 \%$ to the Caribbean and Latin America. Military travel (46\%) and vacation (40\%) were most common reasons for travel. Among them, $20 \%$ took doxycycline, $50 \%$ other prophylaxis, and $30 \%$ took none; 8.7\% developed ILI. Decreased RR of ILI was associated with doxycycline (RR 0.65 [0.43-0.99], $P=0.046)$ and military travel (RR 0.30 [0.21-0.43], $P<0.01)$. Increased risk of ILI was associated with female gender (RR 1.57 [1.24-1.98], $P<$ 0.01 ), travel to Asia (RR 1.37 [1.08-1.75], $P=0.01$ ), and cruises (RR 2.21 [1.73-2.83], $P<0.01$ ). Use of doxycycline malaria prophylaxis is associated with a decreased risk of ILI. Possible reasons include anti-inflammatory or antimicrobial effects, or other unmeasured factors. With few strategies for decreasing ILI in travelers, these findings bear further investigation.
\end{abstract}

\section{INTRODUCTION}

International travel for leisure, business, and/or military activities is increasingly common. With increased travel, there is increased risk of travel-related illness such as malaria, traveler's diarrhea, and influenza-like illness (ILI). According to the 2011 GeoSentinel surveillance data, 23.3\% of travelers reported a febrile illness. Of those febrile travelers, $29 \%$ were diagnosed with malaria and $10.9 \%$ were diagnosed with an ILI. ${ }^{1}$ Prevention of travel-related illness involves pretravel counseling, immunizations, and chemoprophylaxis. The mainstay of ILI prevention is hand hygiene and influenza vaccination, ${ }^{2}$ although the efficacy of these in preventing travel-related ILI is unknown.

Doxycycline is commonly used for malaria prophylaxis among international travelers. It is known to have activity against numerous respiratory pathogens, several pathogens associated with fever in the returning traveler, and has general anti-inflammatory effects but has not been evaluated to our knowledge for an association with development of ILI in travelers. ${ }^{3}$ In a cohort of travelers using a variety of malaria preventive strategies, we sought to evaluate whether the use of doxycycline as malaria prophylaxis was associated with a decreased risk of subjective ILI during international travel.

\section{MATERIALS AND METHODS}

Study design. This study was a subset of the larger TravMil study: Deployment and Travel Related Infectious Disease Risk Assessment, Outcomes, and Prevention Strategies Among

\footnotetext{
*Address correspondence to Kathryn Lago, Infectious Disease Service, Brooke Army Medical Center, 3551 Roger Brooke Dr., Joint Base San Antonio-Fort Sam Houston, Fort Sam Houston, TX 78234. E-mail: kathryn.j.lago.mil@mail.mil
}

Department of Defense $(D o D)$ Beneficiaries, approved by the Uniformed Services University of the Health Sciences Institutional Review Board (Bethesda, MD). TravMil is a prospective observational cohort of DoD beneficiaries traveling outside the continental United States for $\leq 6.5$ months. Consenting adults were enrolled pretravel at six military travels (Walter Reed National Military Medical Center, Bethesda, MD; Brooke Army Medical Center, San Antonio, TX; Naval Medical Center, Portsmouth, VA; Madigan Army Medical Center, Tacoma, WA; Naval Medical Center, San Diego, CA; and Landstuhl Regional Medical Center, Landstuhl, Germany) and in the pre-deployment setting between January 2010 and August 2018. Eligible travelers were limited to DoD beneficiaries (e.g., active duty military personnel, military retirees, USPHS, employees of Department of State, and militarydependent family members). Individuals were required to be traveling outside of the continental United States, Puerto Rico, Guam, Samoa, the U.S. Virgin Islands, Western or Northern Europe, Canada, or New Zealand for at least part of their itinerary.

Survey data. Pretravel enrollees completed a pretravel survey recording their demographics, travel characteristics, and prescriptions for malaria prophylaxis. Travelers were also provided a diary to record episodes of fever, cough, and sore throat during travel. Subjective ILI was defined as a subjective fever with either a sore throat or cough. Episodes of subjective ILI were analyzed for each registered trip. For each registered trip which could include multiple geographic regions, the traveler was prescribed doxycycline, other, or no malaria prophylaxis for the duration of that trip.

Statistical analysis. Univariate and multivariate analysis were performed to determine which trip and traveler characteristics, including use of malaria prophylaxis, were associated with development of subjective ILI. For univariate and 
multivariate analysis, a Poisson regression with robust error variance was used to estimate the relative risk (RR) of our count data. A robust error variance allowed for a tighter $\mathrm{Cl}$. Variables with a $P$-value of $\leq 0.05$ on univariate analysis underwent a Poisson regression for multivariate analysis to assess independent risk factors. These criteria allowed for exclusion of highly correlated covariate and mutually exclusive variables. Results of multivariate analysis were reported with RR with $95 \%$ Cls. Statistical significance was defined as a $P$-value of $\leq 0.05$. Statistical analysis was performed with SPSS software (IBM SPSS Statistics Version 22, Chicago, IL).

\section{RESULTS}

Baseline characteristics. A total of 3,227 trips with 2,803 travelers were analyzed (Table 1). The majority of travelers were male $(62.1 \%)$ with a median age of 39 years (IQR 27, 59) and with a median duration of travel of 19 days (IQR 12, 49). Caucasian $(70.7 \%)$ was the most commonly reported race. Twenty-five percent of travelers were $\geq 60$ years old and $39 \%$ who developed subjective ILI were $\geq 60$ years old. For malaria prophylaxis, $20 \%$ were prescribed doxycycline, $50 \%$ prescribed another form of malaria prophylaxis, and $30 \%$ were prescribed no malaria prophylaxis. Of the other malaria prophylaxis group, 89\% were atovaquone-proguanil. Travelers' itineraries could include more than one region of travel. Forty percent traveled to Asia, 32\% traveled to Africa, 28\% traveled to the Caribbean, Mexico, Central America, and South America. The cumulative incidence of subjective ILI was $9 \%$ with an incidence rate of 26.5 per 1,000 travel days.

More than one purpose of travel and multiple types of travel accommodations could be reported (Table 2). The most common purposes of travel were military (46\%) and vacation (40\%). Only $3 \%$ of travelers who traveled for military purposes were nonactive duty (representing government employees/contractors). The most common travel accommodation was hotel $(71 \%)$. Eleven percent of travelers went on a cruise. Seventeen percent of those cruise ship travelers were traveling for military purpose.
Statistical analysis. On univariate analysis of travel characteristics (Table 3), female travelers, travelers in Asia, South America, and longer duration of travel were associated with increased risk of developing subjective ILI. Vacation, visiting friends and relatives, and cruise ship travel were associated with increased risk of developing subjective ILI. On univariate analysis, use of doxycycline for malaria prophylaxis (compared with the use of other or no prophylaxis, combined to optimize sample size), travel to Africa, and military purpose of travel were associated with decreased risk of subjective ILI. In addition to doxycycline, gender, military purpose of travel, travel to Asia, cruise ship travel, and duration of travel were chosen as variables for multivariate analysis. Gender and cruise ship travel were selected because they had a strong effect on the univariate analysis and there have been several published studies on these variables and ILI rates. Military purpose of travel was chosen as this is one of the key populations in the TravMil group and to ensure that doxycycline use and military travel were both considered in the model simultaneously. Travel to Asia was chosen for multivariate analysis as it represents the largest area of travel in our study, and was also significantly associated with ILI on univariate analysis. On multivariate analysis (Table 4), use of doxycycline (0.65 [0.43-0.99], $P=0.046)$ and military purpose of travel $(0.3$ [0.21-0.43], $P<0.01)$ remained significantly associated with decreased risk. On multivariate analysis, female gender (1.57 [1.24-1.98], $P<0.01)$, median duration of travel in days (1.0 [1.0-1.01], $P<0.01)$, travel to Asia (1.37 [1.08-1.75], $P=0.01)$, and cruise ship travel (2.21 [1.73-2.83], $P<0.01)$ were associated with increased risk.

Military subgroup analysis. Because $82 \%$ of the doxycycline group were traveling for a military purpose, a subgroup analysis was performed (Table 5). A multivariate Poisson (performed with the same variables as aforementioned, with the exception of military purpose of travel because this was the subset being analyzed, and with the addition of Africa because of this being the second most common region of deployment and also significant on univariate analysis)

TABLE 1

Traveler demographics, malaria prophylaxis prescribed, destinations, and incidence of ILI

\begin{tabular}{|c|c|c|c|}
\hline Characteristics & All $(N=3,227)$ & Non-deployment travel $(N=2,154)$ & Deployment $(N=1,073)$ \\
\hline Gender, male & $2,004(62 \%)$ & $1,106(52 \%)$ & $898(84 \%)$ \\
\hline Median age (years) & 39 (IQR 27,59) & 51 (IQR 33,65) & 28 (IQR 23,35) \\
\hline Median duration of travel (days) & 19 (IQR 12,49) & 16 (IQR 11,27) & 34 (IQR 16, 84) \\
\hline \multicolumn{4}{|l|}{ Race } \\
\hline African American & $345(11 \%)$ & $244(11 \%)$ & $101(9 \%)$ \\
\hline Caucasian & $2,252(71 \%)$ & 1,508 (70\%) & $744(69 \%)$ \\
\hline \multicolumn{4}{|l|}{ Military status } \\
\hline Active duty & $1,658(51 \%)$ & $585(27 \%)$ & $1,073(100 \%)$ \\
\hline Dependent & $1,454(45 \%)$ & $1,454(68 \%)$ & - \\
\hline \multicolumn{4}{|l|}{ Malaria prophylaxis prescribed } \\
\hline Doxycycline & $644(20 \%)$ & $174(8 \%)$ & $470(44 \%)$ \\
\hline Other $^{*}$ & $1,623(50 \%)$ & $1,235(57 \%)$ & $388(36 \%)$ \\
\hline None & $960(30 \%)$ & 745 (35\%) & 215 (20\%) \\
\hline \multicolumn{4}{|l|}{ Destinations } \\
\hline Africa & $1,033(32 \%)$ & $661(31 \%)$ & $372(35 \%)$ \\
\hline Asia & $1,299(40 \%)$ & $752(35 \%)$ & 547 (51\%) \\
\hline $\begin{array}{l}\text { Caribbean, Mexico, and Central } \\
\text { America }\end{array}$ & $704(22 \%)$ & $583(27 \%)$ & $121(11 \%)$ \\
\hline South America & $356(11 \%)$ & $332(15 \%)$ & $24(2 \%)$ \\
\hline Multiple regions & $1,013(31 \%)$ & 519 (24\%) & $494(46 \%)$ \\
\hline ILI cumulative incidence & $281(9 \%)$ & $265(12 \%)$ & $16(2 \%)$ \\
\hline
\end{tabular}

II = influenza-like illness.

* $89 \%$ atovaquone-proguanil. 
TABLE 2

Purpose of travel, travel accommodations, and type of travel location

\begin{tabular}{lccc}
\hline \multicolumn{1}{c}{ Characteristic } & All $(N=3,227)$ & Non-deployment travel $(N=2,154)$ & Deployment $(N=1,073)$ \\
\hline Purpose of travel & & & \\
$\quad$ Military & $1,468(46 \%)$ & $395(18 \%)$ & $1,073(100 \%)$ \\
Vacation & $1,282(40 \%)$ & $1,282(40 \%)$ & - \\
Visiting friends and relatives & $386(12 \%)$ & $386(12 \%)$ & - \\
$\quad$ Medical & $148(5 \%)$ & $148(5 \%)$ & - \\
Travel accommodations & & & $4,814(84 \%)$ \\
$\quad$ Hotel & $2,295(71 \%)$ & $314(15 \%)$ & $40(4 \%)$ \\
Cruise & $354(11 \%)$ & $198(6 \%)$ & - \\
Military accommodations & $198(6 \%)$ & $398(12 \%)$ & - \\
Private accommodations & $398(12 \%)$ & $1,036(48 \%)$ & $716(67 \%)$ \\
Type of location & & & \\
$\quad$ Rural & $1,752(54 \%)$ & & \\
\hline
\end{tabular}

showed that doxycycline continued to have a statistically significant decreased RR of subjective ILI (0.42 [0.19-0.91], $P=0.03)$. Female gender (2.43 [1.39-4.25], $P<0.01)$ and cruise ship travel (4.6 [2.28-9.26], $P<0.01$ ) continued to have a statistically significant increased RR of subjective ILI.

\section{DISCUSSION}

Our evaluation of the use of doxycycline as malaria prophylaxis and risk of subjective ILI among international travelers is to our knowledge the only such report in the medical literature and demonstrates a negative association between doxycycline used as malaria prophylaxis and incidence of subjective ILI in international travelers. Given the widespread prevalence of ILI in international travelers, as well as the limitations and unclear efficacy of the currently recommended interventions for prevention in this context, these findings warrant consideration. Although these observational data cannot be used to determine causality, both antimicrobial and anti-inflammatory effects of the drug suggest the possibility of a biologically plausible effect. Because there are limited data on chemoprophylaxis strategies for ILI and the effect of doxycycline on the microbiology of ILI, we explored literature of uses of doxycycline for prevention of lower respiratory disease.

Empiric uses of doxycycline for prophylaxis or treatment of respiratory infection have been recommended outside of travel medicine. The use of doxycycline for prophylaxis in patients with chronic obstructive pulmonary disease (COPD), to decrease the frequency of exacerbations and improve quality of life, has been well described. ${ }^{4}$ In 2007, the British Thoracic Society published guidelines for treatment on ILI during an influenza pandemic. They recommended empiric use of doxycycline or amoxicillin-clavulanate for bacterial pneumonia in COPD or severe preexisting illness. ${ }^{5}$ During the 2009 H1N1 pandemic, the United Kingdom Department of Health re-emphasized the use of empiric antibiotics for highrisk patients presenting with ILI. ${ }^{6}$ Further analysis of patients

TABLE 3

Univariate analysis of characteristics and their RR of ILI

\begin{tabular}{|c|c|c|c|c|}
\hline Characteristic & ILI & No ILI & Univariate RR $(95 \% \mathrm{Cl})$ & Univariate $P$-value \\
\hline \multicolumn{5}{|l|}{ Malaria prophylaxis } \\
\hline Doxycycline & $29(5 \%)$ & $615(95 \%)$ & $0.46(0.32-0.67)$ & $<0.01$ \\
\hline Other or none & $252(10 \%)$ & $2,331(90 \%)$ & Ref & \\
\hline Other & $144(9 \%)$ & $1,479(91 \%)$ & $0.78(0.62-0.99)$ & \\
\hline None & $108(11 \%)$ & $852(89 \%)$ & Ref & \\
\hline \multicolumn{5}{|l|}{ Gender } \\
\hline Male & $121(6 \%)$ & $1,885(94 \%)$ & Ref & \\
\hline Female & $160(12 \%)$ & $1,061(88 \%)$ & $2.16(1.73-2.72)$ & $<0.01$ \\
\hline Age (years, IQR) & $54(31,66)$ & $38(27,57)$ & - & $<0.01$ \\
\hline Median duration of travel (days, IQR) & $21(14,36)$ & $19(12,42)$ & - & 0.03 \\
\hline \multicolumn{5}{|l|}{ Destinations } \\
\hline Africa & $75(7 \%)$ & $958(93 \%)$ & $0.77(0.60-0.99)$ & 0.05 \\
\hline Asia & $133(10 \%)$ & 1,166 (90\%) & $1.26(1.00-1.58)$ & 0.04 \\
\hline South America & $46(14 \%)$ & $286(86 \%)$ & $1.70(1.27-2.29)$ & 0.04 \\
\hline Mexico/Caribbean/Central America & $53(9 \%)$ & $557(91 \%)$ & $0.99(0.75-1.32)$ & 0.99 \\
\hline Multiple regions & $93(9 \%)$ & 920 (91\%) & $1.08(0.85-1.37)$ & 0.52 \\
\hline \multicolumn{5}{|l|}{ Purpose of travel } \\
\hline Military & $53(4 \%)$ & $1,415(96 \%)$ & $0.28(0.20-0.37)$ & $<0.01$ \\
\hline Vacation & $176(22 \%)$ & $1,106(78 \%)$ & $2.54(2.02-3.20)$ & $<0.01$ \\
\hline Visiting friends and relative & $46(12 \%)$ & $340(88 \%)$ & $1.44(1.06-1.94)$ & 0.02 \\
\hline Medical & $10(7 \%)$ & $138(3 \%)$ & $0.76(0.42-1.41)$ & 0.39 \\
\hline \multicolumn{5}{|l|}{ Travel accommodations } \\
\hline Hotel & $180(10 \%)$ & $1,666(90 \%)$ & $1.33(1.06-1.68)$ & 0.02 \\
\hline Cruise $^{\star}$ & $73(21 \%)$ & $281(79 \%)$ & $2.84(2.23-3.63)$ & $<0.01$ \\
\hline Military & $20(10 \%)$ & $178(90 \%)$ & $1.17(0.76-1.80)$ & 0.47 \\
\hline
\end{tabular}

ive risk

* Including deployment on USS comfort. 
TABLE 4

Multivariate analysis of characteristics and their RR of influenza-like illness

\begin{tabular}{lcc}
\hline \multicolumn{1}{c}{ Characteristic } & Multivariate RR $(95 \% \mathrm{Cl})$ & Multivariate $P$-value \\
\hline Doxycycline & $0.65(0.43-0.99)$ & 0.046 \\
Female & $1.57(1.24-1.98)$ & $<0.01$ \\
Median duration of travel (days) & $1.01(1.0-1.01)$ & $<0.01$ \\
Asia & $1.37(1.08-1.75)$ & 0.01 \\
Military travel & $0.30(0.21-0.43)$ & $<0.01$ \\
Cruise & $2.21(1.73-2.83)$ & $<0.01$ \\
\hline \multicolumn{1}{l}{ RR $=$ relative risk. } & &
\end{tabular}

presenting with post-influenza bacterial pneumonia to outpatient care centers throughout the United Kingdom from 2007 to 2010 showed that $96 \%$ of bacterial respiratory isolates were susceptible to doxycycline. ${ }^{7}$ According to the Alexander Project, a large multinational surveillance study of respiratory pathogens, $71.3 \%$ of Streptococcus pneumoniae, and $95.8 \%$ of Moraxella catarrhalis are susceptible to doxycycline. ${ }^{8}$ The Infectious Disease Society of America recommends use of doxycycline as an alternate monotherapy for community-acquired pneumonia for healthy individuals who are not at risk of having drug-resistant Streptococcus pneumoniae infections. ${ }^{9}$ Doxycycline has been shown to have a wide empiric coverage of bacterial respiratory pathogens, which could explain the decreased RR of developing subjective ILI as seen in our study, to the extent that bacterial pathogens contribute to ILI, which is unclear.

Specific bacterial causes of respiratory infection in international travelers are less well defined, and it is challenging to know what proportion of these may be covered by doxycycline. Previous studies have reported influenza rates among returning travelers to be from 6 to $30 \%{ }^{10,11}$ and streptococcal pharyngitis rates around $6 \% .{ }^{10}$ However, some studies with ILI in the returning traveler only look for viral pathogens as the etiology of their respiratory symptoms. ${ }^{11,12}$ GeoSentinel, which only captures those travelers who are ill enough after travel to seek medical care and records specific diagnoses as they have been recorded in the course of usual clinical care, reports only 35 cases of Legionella, 30 cases of pertussis, and two cases of diphtheria out of its 600 travelers reporting respiratory illnesses. ${ }^{1}$ With these reports of bacterial pathogens as the cause of respiratory illness in returning travelers, it is microbiologically plausible that doxycycline may be covering some of these pathogens.

The military is a unique population of travelers who may be at higher risk for acquiring respiratory pathogens as they stay in crowded accommodations, interact with local populations, and may have limited sanitation. In a retrospective study, deployers to Operation Enduring Freedom had pre- and post-

TABLE 5

Multivariate analysis of characteristics and their RR of influenza-like illness in military subgroup

\begin{tabular}{lcr}
\hline \multicolumn{1}{c}{ Characteristic $(N=1,468)$} & Multivariate RR $(95 \% \mathrm{Cl})$ & $P$-value \\
\hline Doxycycline & $0.42(0.19-0.91)$ & 0.03 \\
Female & $2.43(1.39-4.25)$ & $<0.01$ \\
Median duration of travel (days) & $1.0(0.99-1.01)$ & 0.10 \\
Asia & $1.2(0.57-2.5)$ & 0.65 \\
Africa & $1.2(0.54-2.71)$ & 0.63 \\
Cruise & $4.6(2.28-9.26)$ & $<0.01$ \\
\hline RR $=$ relative risk. &
\end{tabular}

$\mathrm{RR}=$ relative risk. deployment sera taken to look for rates of respiratory pathogen seroconversion. One of seven deploying personnel seroconverted to a respiratory pathogen. Of note, there was a $10 \%$ seroconversion rate to Chlamydia pneumoniae, $4.3 \%$ conversion rate to Bordetella pertussis, and $3.7 \%$ conversion rate to Mycoplasma pneumoniae. The seroconversion rate of $B$. pertussis is two to four times higher than the adult U.S. population. ${ }^{13}$ In addition, GeoSentinel showed that respiratory bacterial infections such as streptococcal pharyngitis, sinusitis, and otitis media were significantly greater in younger persons with median ages in the 20-30s, which is similar to our study population. ${ }^{10}$ Therefore, our study population could be experiencing a slightly higher rate of bacterial pathogens than the normal travel population. Of importance, GeoSentinel clinics use multiple diagnostic methods to determine etiologic agents of disease, and our study lacks pathogen identification. In addition, GeoSentinel clinics capture travelers presenting to their clinic at the time of their acute illness. However, most travelers with ILI will have their illness resolve before being evaluated at a travel clinic, as was the case in our study. Therefore, it is difficult to truly extrapolate the GeoSentinel population to in our study. Furthermore, it is challenging to determine if the antimicrobial properties are contributing to the decreased RR of subjective ILI seen in our study. Importantly, doxycycline has been used in medicine solely for its anti-inflammatory properties.

Tetracyclines have a regulatory influence on the immune system and inflammatory pathways. ${ }^{14,15}$ Doxycycline has been shown to inhibit activation of macrophages which in turn inhibits tumor necrosis factor (TNF)- $\alpha$, interleukin (IL) 1b, IL-6, and other pro-inflammatory proteins. Tetracyclines also inhibit lipopolysaccharide-induced nitric oxide production. Nitric oxide production has been implicated in the pathophysiology of sepsis and rheumatoid arthritis. ${ }^{14}$ Doxycycline is currently being studied for its anti-inflammatory properties to treat allergeninduced inflammation, osteoarthritis, and corneal inflammation. Doxycycline also has anti-oxidant properties, thereby reducing tissue destruction. ${ }^{16}$ Therefore, the decreased RR of subjective ILI seen in our study could be related to the anti-inflammatory properties associated with doxycycline.

Several additional factors described in this study as being associated with the risk of ILI are consistent with previously published data. Associations with gender and the development of ILI, as well as the causes of ILI have been previously reported. ${ }^{10,17,18}$ A study of military travelers to Iraq and Afghanistan showed an increased RR of developing ILI in females. ${ }^{17}$ Similarly, GeoSentinel data have shown a statistically significant decreased odds ratio (OR) of developing upper respiratory infections in males. However, there was an increased OR of developing lower respiratory tract infection and pneumonia in males. ${ }^{10}$

Cruise ship travel has also been described for being associated with increased risk of acquiring an ILI among travelers. ${ }^{10,19}$ Pavli et al. described different respiratory pathogens acquired by cruise ship passengers and crew in a 3 -year prospective study. The ILI attack rate was $0.6 \%$ among passengers and $1.3 \%$ among crew members. There were no reported cases of influenza A or B. However, rapid Streptococcus testing was positive in $4.5 \%$ of passengers and $30.9 \%$ of crew members. ${ }^{19}$

Our study had multiple limitations. First, our study collected observational data with self-reported ILI and subjective fever. 
Collecting subjective self-reported data introduces recall bias, and lack of pathogen identification makes it difficult to determine if the travelers truly had an ILI. In addition to lack of pathogen identification, it is not possible to know whether the decreased RR of subjective ILI with doxycycline could be due to its antimicrobial properties. Giving travelers a thermometer and real-time methods of collecting sputum and/or blood samples for polymerase chain reaction (PCR) could have allowed for more robust data to determine the association of reduced risk of ILI and doxycycline. In addition, because this is observational data, there may be potential unmeasured confounders. Some of those confounders include the possibility of unrecorded chronic diseases in nonmilitary members such as underlying cardiac or respiratory illnesses, smoking history, household contacts with respiratory illnesses in the week before travel, the effects of living or working in congregate settings, and the season of infection. An additional limitation to this study is that pneumococcal vaccination status and antibiotic use before or during travel were not analyzed.

Influenza season is dependent on geographic location. In our study, median travel duration was 19 days. However, on military deployment trips, the median duration was 34 days. In addition, subjects frequently traveled to multiple locations, $31 \%$, and therefore, portions of their travel could be in different regions of influenza exposure. Because so many trips involved changes in season (including changes in seasonality based on geography), we did not explore seasons of travel and their effect on subjective ILI.

Influenza immunization and hand hygiene are recommended by the CDC to prevent ILI in international travelers. ${ }^{2}$ Active military personnel are required to receive annual influenza vaccination. Therefore, decreased rates of subjective ILI among military members may be secondary to immunization status. Previous TravMil studies ${ }^{20}$ have shown that there was no statistically significant effect on ILI with increasing number of influenza vaccines over a 5-year period; thus, this variable was not re-explored. This finding is in contrast to previously published data by Qureshi et al., which showed the influenza attack rate was lower among pilgrims per Haji who had influenza vaccine (attack rate of $36 \%$ versus $62 \%$, respectively). However, in the Qureshi et al. ${ }^{21}$ study group, only $54 \%$ of subjects had pretravel vaccines.

One of the greatest strengths of our study include its large sample size with 3,227 trips recorded and including various types of malaria prophylaxis taken, which allowed for statistical analysis to compare the effects of doxycycline for prophylaxis versus other or no prophylaxis on subjective ILI incidence. Furthermore, our military subgroup analysis showed that use of doxycycline was still associated with a statistically significant decrease RR of subjective ILI, further strengthening our findings and decreasing the likelihood that the decrease in subjective ILI risk seen with doxycycline use may be attributed to some other military-specific aspect of travel.

\section{CONCLUSION}

The cumulative incidence of subjective ILI in international travelers who were taking doxycycline for malaria prophylaxis was decreased compared with those taking other or no prophylaxis. This effect may be secondary to the multiple properties of doxycycline including, but not limited to: antibiotic, antiviral, anti-inflammatory, and anti-oxidant effects. The mainstay of ILI management in a traveler is prevention with good hand hygiene and influenza vaccine. ${ }^{2}$ With few other strategies known to decrease ILI, further investigation on the correlation between doxycycline use and incidence of ILI is warranted.

Received August 30, 2019. Accepted for publication November 12, 2019.

Published online February 10, 2020.

Acknowledgments: We thank the Infectious Disease Clinical Research Program TravMil study team of clinical coordinators, laboratory technicians, data managers, clinical site managers, and administrative support personnel for their contributions to this project.

Financial support: This study (IDCRP-037) was supported by the Infectious Disease Clinical Research Program (IDCRP), a Department of Defense (DoD) program executed by the Uniformed Services University of the Health Sciences (USUHS) through a cooperative agreement with The Henry M. Jackson Foundation for the Advancement of Military Medicine, Inc. (HJF). This project has been funded in whole, or in part, with federal funds from the National Institute of Allergy and Infectious Diseases, National Institutes of Health $(\mathrm{NIH})$, under Inter Agency Agreement Y1-Al-5072 and from the Department of Defense Global Emerging Infections Surveillance.

Copyright statement: Some authors are service members of the U.S. Government. This work was prepared as part of their official duties. Title 17 U.S.C. $\$ 105$ provides that "Copyright protection under this title is not available for any work of the United States Government." Title 17 U.S.C. $\$ 101$ defines a U.S. Government work as a work prepared by a military service member or by an employee of the U.S. Government as part of that person's official duties.

Disclaimer: The contents of this publication are the sole responsibility of the author(s) and do not necessarily reflect the views, opinions, or policies of Uniformed Services University of the Health Sciences (USUHS), the Department of Defense (DoD), the Departments of the Army, Navy, or Air Force, or Brooke Army Medical Center, the U.S. Army Medical Department, the U.S. Army Office of the Surgeon General, Landstuhl Regional Medical Center, Walter Reed National Military Medical Center, Madigan Army Medical Center, Naval Medical Center Portsmouth, or The Henry M. Jackson Foundation for the Advancement of Military Medicine, Inc. The views expressed in this manuscript are those of the author (s) and do not reflect the official policy or position of the Department of the Army, Department of Defense, or the U.S. Government. Mention of trade names, commercial products, or organizations does not imply endorsement by the U.S. Government. The investigators have adhered to the policies for protection of human subjects as prescribed in 45 CFR 46.

Authors' addresses: Kathryn Lago and Heather Yun, Brooke Army Medical Center, Fort Sam Houston, TX, and Uniformed Services University of the Health Sciences, Bethesda, MD, E-mails: kathryn.j.lago.mil@mail.mil and heather.c.yun.mil@mail.mil. Kalyani Telu, Department of Preventive Medicine and Biostatistics, Infectious Disease Clinical Research Program, Uniformed Services University of the Health Sciences, Bethesda, MD, and The Henry M. Jackson Foundation for the Advancement of Military Medicine, Inc., Bethesda, MD, E-mail: ktelu@idcrp.org. David Tribble, Department of Preventive Medicine and Biostatistics, Infectious Disease Clinical Research Program, Uniformed Services University of the Health Sciences, Bethesda, MD, E-mail: david.r.tribble.civ@mail.mil. Anuradha Ganesan, Department of Preventive Medicine and Biostatistics, Infectious Disease Clinical Research Program, Uniformed Services University of the Health Sciences, Bethesda, MD, Walter Reed National Military Medical Center, Bethesda, MD, and The Henry M. Jackson Foundation for the Advancement of Military Medicine, Inc., Bethesda, MD, E-mail: anuradha.ganesan.ctr@mail.mil. Anjali Kunz, Madigan Army Medical Center, Tacoma, WA, E-mail: anjali.n.kunz.mil@mail.mil. Charla Geist, Landstuhl Regional Medical Center, Landstuhl, Germany, E-mail: charla.c.geist.mil@mail.mil. Jamie Fraser and Indrani Mitra, Department of Preventive Medicine and Biostatistics, Infectious Disease Clinical Research Program, Uniformed Services University of the Health Sciences, Bethesda, MD, and The Henry M. Jackson 
Foundation for the Advancement of Military Medicine, Inc., Bethesda, MD, E-mail: jamie.fraser.ctr@usuhs.edu and imitra@idcrp.org. Tahaniyat Lalani, Department of Preventive Medicine and Biostatistics, Infectious Disease Clinical Research Program, Uniformed Services University of the Health Sciences, Bethesda, MD, The Henry M. Jackson Foundation for the Advancement of Military Medicine, Inc., Bethesda, MD, and Naval Medical Center, Portsmouth, VA, E-mail: tlalani@idcrp.org.

This is an open-access article distributed under the terms of the Creative Commons Attribution (CC-BY) License, which permits unrestricted use, distribution, and reproduction in any medium, provided the original author and source are credited.

\section{REFERENCES}

1. Leder K et al., 2013. GeoSentinel surveillance of illness in returned travelers, 2007-2011. Ann Intern Med 158: 456-468.

2. Goeijenbier M, van Genderen $P$, Ward BJ, Wilder-Smith $A$, Steffen $R$, Osterhaus $A D, 2016$. Travellers and influenza: risks and prevention. J Travel Med 24: 1-10.

3. Thwaites GE, Day NPJ, 2017. Approach to fever in the returning traveler. N Engl J Med 376: 548-560.

4. Herath SC, Poole P, 2013. Prophylactic antibiotic therapy for chronic obstructive pulmonary disease (COPD). Cochrane Database Syst Rev 11: CD009764. Available at: https://doi. org/10.1002/14651858.CD009764.pub2.

5. British Infection Society, British Thoracic Society, Health Protection Agency, 2007. Pandemic flu: clinical management of patients with an influenza-like illness during an influenza pandemic. Thorax 62: 1-46.

6. UK Department of Health, 2009. Pandemic H1N1 2009 Influenza: Clinical Management Guidelines for Adults and Children. Available at: http://www.dh.gov.uk/en/Publicationsandstatistics/Publications/ PublicationsPolicyAndGuidance/DH_107769. Accessed March 22, 2019.

7. Blackburn RM, Henderson KL, Lillie M, Sheridan E, George RC, Deas AHB, Johnson AP, 2011. Empirical treatment of influenzaassociated pneumonia in primary care: a descriptive study of the antimicrobial susceptibility of lower respiratory tract bacteria (England, Wales, and Northern Ireland, January 2007March 2010). Thorax 66: 389-395.

8. Jacobs MR, Felmingham D, Appelbaum PC, Gruneberg RN; the Alexander Project Group, 2003. The Alexander project 1998-2000: susceptibility of pathogens isolated from community-acquired respiratory tract infection to commonly used antimicrobial agents. J Antimicrob Chemother 52: 229-246.

9. Mandell LA et al., 2007. Infectious disease society of America/ American thoracic society consensus guidelines on the management of community-acquired pneumonia in adults. Clin Infect Dis 44 (Suppl 2): S27-S72.

10. Leder K, Sundararajan V, Weld L, Pandey P, Brown G, Torresi J, 2003. Respiratory tract infections in travelers: a review of the GeoSentinel surveillance network. Clin Infect Dis 36: 399-406.

11. Laguna-Torres VA, 2009. Influenza-like illness sentinel surveillance in Peru. PLoS One 4: e6118.

12. Ratnam I et al., 2013. Incidence and risk factors for acute respiratory illnesses and influenza virus infections in Australian travelers to Asia. J Clin Virol 57: 54-58.

13. Eick $A$ et al., 2011. Serosurvey of bacterial and viral respiratory pathogens among deployed U.S. service members. Am J Prev Med 41: 573-580.

14. Dunston CR, Griffiths HR, Lambert PA, Staddon S, Vernallis AB, 2011. Proteomic analysis of the anti-inflammatory action of minocycline. Proteomics 11: 42-51.

15. Di Caprio R, Lembo S, Di Costanzo L, Balato A, Monfrecola G, 2015. Anti-Inflammatory properties of low and high doxycycline doses: an in vitro study. Mediators Inflamm 2015: 329418. Available at: https://doi.org/10.1155/2015/329418. Epub 2015 Apr 22.

16. Bahrami F, Morris DL, Pourgholami MH, 2012. Tetracyclines: drugs with huge therapeutic potential. Mini Rev Med Chem 12: 44-52.

17. Soltis BW, Sanders JW, Putnam SD, Tribble DR, Riddle MS, 2009. Self reported incidence and morbidity of acute respiratory illness among deployed US military in Iraq and Afghanistan. PLoS One 4: e6177.

18. Yun HC, Fugate WH, Murray CK, Cropper TL, Lott L, McDonald $\mathrm{JM}, 2014$. Pandemic influenza virus $2009 \mathrm{H} 1 \mathrm{~N} 1$ and adenovirus in a high risk population of young adults: epidemiology, comparison of clinical presentations, and coinfection. PLoS One 9: e8504.

19. Pavil A, Maltezou HC, Papadakis A, Katerelos P, Saroglou G, Tsakris A, Tsiodras S, 2016. Respiratory infections and gastrointestinal illness on a cruise ship: a three-year prospective study. Travel Med Infect Dis 14: 389-397.

20. Wood S, 2018. Influenza-like Illness in travelers to the developing world. Am J Trop Med Hyg 99: 1269-1274.

21. Qureshi H, Gessner BD, Leboulleux D, Hasan H, Alam SE, Moulton $\mathrm{LH}, 2000$. The incidence of vaccine preventable influenza-like illness and medication use among Pakistani pilgrims to the Haj in Saudi Arabia. Vaccine 18: 2956-2962. 